
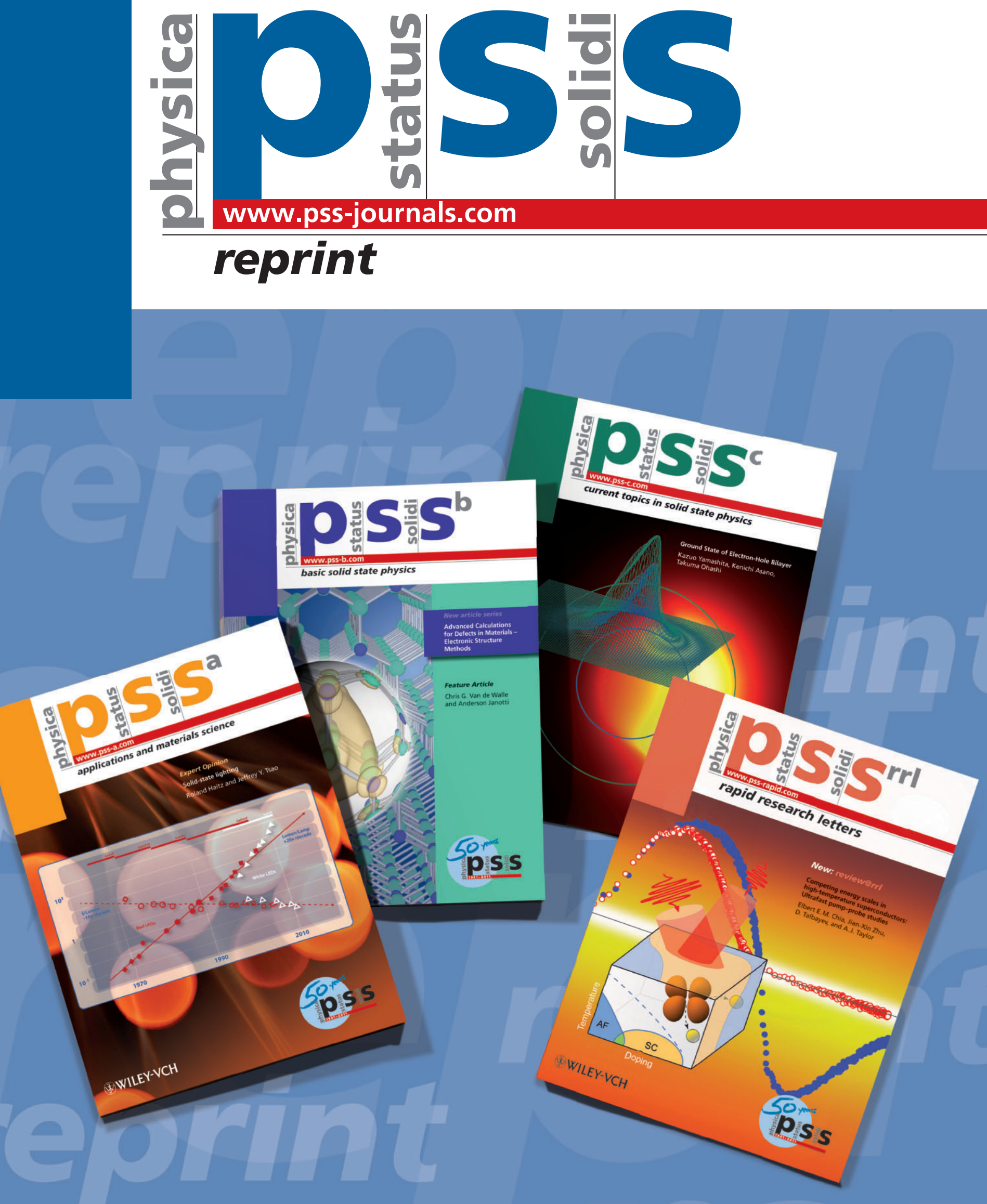


\title{
The source of room temperature ferromagnetism in granular GaMnAs layers with zinc blende clusters
}

\author{
K. Lawniczak-Jablonska*, ${ }^{*}$, J. Libera ${ }^{1}$, A. Wolska', M. T. Klepka', P. Dluzewski ${ }^{1}$, J. Sadowski ${ }^{1,2}$, D. Wasik ${ }^{3}$, \\ A. Twardowski ${ }^{3}$, A. Kwiatkowski ${ }^{3}$, and K. Sato ${ }^{4}$ \\ ${ }^{1}$ Institute of Physics PAS, al. Lotnikow 32/46, 02668 Warsaw, Poland \\ ${ }^{2}$ MAX-Lab, Lund University P.O. Box. 118, 22100 Lund, Sweden \\ ${ }^{3}$ Institute of Experimental Physics, Faculty of Physics, University of Warsaw, ul. Hoza 69, 00681 Warsaw, Poland \\ ${ }^{4}$ Department of Materials Engineering Science, Graduate School of Engineering Science, Osaka University, 1-3 Machikaneyama, \\ Toyonaka, Osaka 560-8531, Japan
}

Received 23 November 2010, accepted 15 December 2010

Published online 20 December 2010

Keywords GaMnAs, semiconductors, granular material, spintronics, room temperature ferromagnetism

${ }^{*}$ Corresponding author: e-mail jablo@ifpan.edu.pl

Granular GaAs: (Mn,Ga)As films were prepared by annealing the $\mathrm{Ga}_{0.985} \mathrm{Mn}_{0.015} \mathrm{As} / \mathrm{GaAs}$ layers at $500{ }^{\circ} \mathrm{C}$ or $600{ }^{\circ} \mathrm{C}$. It is commonly accepted that this processing should result in the formation of cubic or hexagonal MnAs clusters, respectively. We demonstrate that such a priori assumption is not justified. If in the as grown sample there are not many defects with the interstitial $\mathrm{Mn}$ atoms, only small cubic clusters can be formed even after annealing at $600^{\circ} \mathrm{C}$. Moreover, in a sample containing solely cubic GaMnAs clusters, the Mn ions are ferro- magnetically coupled at room temperature. This fact was explained by the existence of GaMnAs solid solution in the clusters, with content of Mn close to $20 \%$ (higher than ever found in the layers) as was confirmed by experiment and theory. Extended X-ray absorption spectroscopy studies excluded the possibility of formation of the hypothetic zinc blende MnAs clusters. Not more than one Mn atom was detected in the second shell around central Mn atom.
1 Introduction The interest in granular materials composed of semiconductor matrix with nanoinclusions of magnetic materials is rapidly growing due to the demonstration of their usefulness in the construction of prototype electronic devices [1]. Large scale applicability of ferromagnetic semiconductors is limited by the Curie temperature, which for GaMnAs layer is below $200 \mathrm{~K}$. In this material the ferromagnetic precipitates are easily produced, yielding multi-phase granular materials as an effect of high temperature annealing at $400-700{ }^{\circ} \mathrm{C}$ of the GaMnAs layers [2, 3] or the Mn-implanted GaAs crystals [4]. It is now commonly accepted that annealing the low temperature grown epilayer at the temperature around $500{ }^{\circ} \mathrm{C}$ produces small cubic clusters of zinc-blende type (ZB) but annealing starting from $600{ }^{\circ} \mathrm{C}$ and higher, results in formation of larger NiAs-type MnAs nanoclusters [2, 3]. Therefore, in many studies, without checking this experimentally, it is a priori assumed that appropriate inclusions have been formed [5]. In this Letter we report the results of studies on the formation of nanoclusters in GaAs/GaMnAs layers in commonly accepted post-annealing conditions and correlate their structure with the ferromagnetism which exists up to room temperature. We also prove that depending on the quality of the as grown layer merely small cubic $(\mathrm{Ga}, \mathrm{Mn})$ As clusters can be formed even after annealing at $600{ }^{\circ} \mathrm{C}$.

2 Results and discussion $\mathrm{Ga}_{0.985} \mathrm{Mn}_{0.015} \mathrm{As}$ layer was grown by molecular beam epitaxy (MBE) on the (001)-oriented GaAs substrate at the temperature of $230{ }^{\circ} \mathrm{C}$. The nominal thickness of the layer was $0.8 \mu \mathrm{m}$. After deposition, the sample was cut into three pieces: one of them was left untreated, the remaining pieces were reintroduced into the $\mathrm{MBE}$ growth chamber and an- 


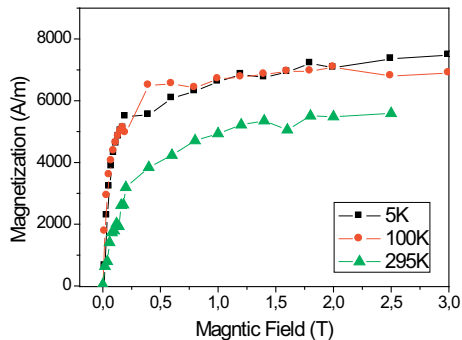

Figure 1 (online colour at: www.pss-rapid.com) Magnetization of $\mathrm{Ga}_{0.985} \mathrm{Mn}_{0.015} \mathrm{As}$ annealed at $600{ }^{\circ} \mathrm{C}$ as a function of magnetic field measured at $5 \mathrm{~K}, 100 \mathrm{~K}$ and $295 \mathrm{~K}$.

nealed under $\mathrm{As}_{2}$ flux for $30 \mathrm{~min}$ at $T=500{ }^{\circ} \mathrm{C}$ and at $T=600{ }^{\circ} \mathrm{C}$.

Magnetization measurements were performed using superconducting quantum interference device (SQUID) magnetometer as a function of temperature $(5-325 \mathrm{~K})$ at constant magnetic fields and at chosen temperatures in the magnetic field from $0 \mathrm{~T}$ to $3.0 \mathrm{~T}$. Magnetic field was applied in-plane of the sample surface. Magnetization data were corrected for the diamagnetic contribution of the GaAs substrate. For annealed samples the magnetization was observed at all temperatures and also at room temperature. The results obtained for the sample annealed at $600{ }^{\circ} \mathrm{C}$ are presented in Fig. 1 . One can notice that from $5 \mathrm{~K}$ to $100 \mathrm{~K}$ the magnetization did not change reaching the saturation at about $1 \mathrm{~T}$. To find the source of the observed room temperature ferromagnetism in the samples a detailed characterization of the sample structure was performed.

Transmission electron microscopy (TEM) studies were performed using a JEOL 2000EX instrument operating at $200 \mathrm{kV}$ accelerating voltage. To get information about the size distribution of clusters the histograms of nanoclusters were acquired from the high-resolution images obtained in $\langle 011\rangle$ projection. It was found that small clusters with cubic ZB structure and sizes less than $10 \mathrm{~nm}$ are present in both samples. In Fig. 2 the example of a TEM image for the sample annealed at $600{ }^{\circ} \mathrm{C}$ is presented together with the related diffraction pattern.

Histograms of the size distribution of clusters are presented in Fig. 3. One can notice that thermal processing at higher temperature does not produce clusters with dimension larger than $7 \mathrm{~nm}$.
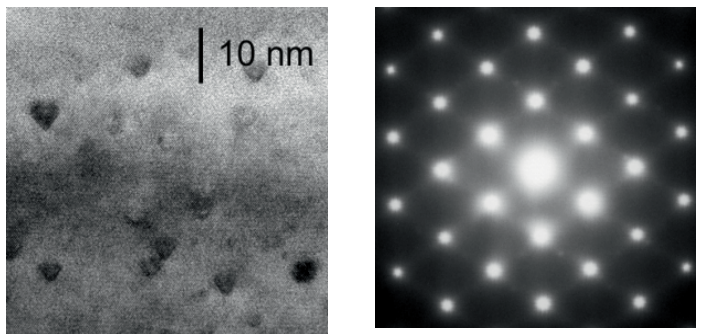

Figure 2 Cross-sectional bright-field TEM image (left) and electron diffraction pattern (right) of granular layer obtained after annealing at $600{ }^{\circ} \mathrm{C}$.
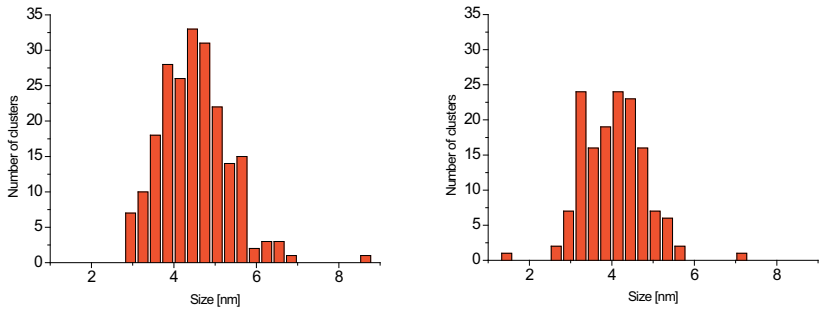

Figure 3 (online colour at: www.pss-rapid.com) Histograms of $\mathrm{Ga}_{0.985} \mathrm{Mn}_{0.015} \mathrm{As}$ sample annealed at $500{ }^{\circ} \mathrm{C}$ (left) and $600{ }^{\circ} \mathrm{C}$ (right).

To examine the local atomic order around $\mathrm{Mn}$ atoms and to confirm the formation of MnAs ZB clusters only, the X-ray absorption spectroscopy (XAS) was applied.

XAS measurements were performed at liquid nitrogen temperature, at the A1 experimental station in DESYHasylab using a double crystal $\mathrm{Si}(111)$ monochromator. The Mn K-edge spectra were registered using a sevenelement fluorescence Si detector. The XAS spectra were analyzed using the IFEFFIT package [7]. The data were analyzed from $k_{\min }=1.9 \AA^{-1}$ to $k_{\max }=12.5 \AA^{-1}$. The fitting was performed in $R$ space. The data were weighted by $k^{3}$ to enhance the oscillations for high $k$.

As a starting model of $\mathrm{ZB}$ clusters it was assumed that the distance of the first coordination sphere is equal to $2.55 \AA[6]$. This value was then refined in the fitting procedure. According to the atomic order in hypothetical ZB MnAs, widely discussed in the literature, Mn atoms should have 4 As atoms in the first shell and in the next shells $12 \mathrm{Mn}$ and $12 \mathrm{As}$ atoms. Surprisingly, for none of the investigated samples $12 \mathrm{Mn}$ atoms can be found in the second coordination sphere. Only Ga atoms in the second sphere provided physical parameters of the fit. The amplitude and phase of scattering photoelectrons remarkably differ for $\mathrm{Mn}$ and $\mathrm{Ga}$ atoms and can be easily distinguished during the EXAFS analysis. Moreover, in the MnAs of the NiAs-type 6 As atoms are present in the first shell followed by $2 \mathrm{Mn}$ and $6 \mathrm{Mn}$ atoms, therefore the existence of such clusters would will be easily discovered in EXAFS analysis.

Concluding, we can rule out the existence of hypothetic pure MnAs nanoclusters in the investigated samples.

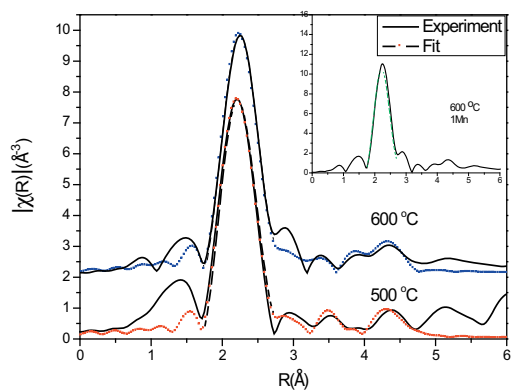

Figure 4 (online colour at: www.pss-rapid.com) $\mathrm{Ga}_{0.985} \mathrm{Mn}_{0.015} \mathrm{As}$ annealed at $500{ }^{\circ} \mathrm{C}$ and $600^{\circ} \mathrm{C}$. Model of (Mn,Ga)As ZB inclusions with $12 \mathrm{Ga}$ in second shell. Inset: Model with $1 \mathrm{Mn}$ and $11 \mathrm{Ga}$ in second shell. Fourier Transform of the EXAFS (full line) and the result of fitting (squares). 
Table 1 Numerical values of EXAFS fitting procedure. $\sigma^{2}$ denotes disorder factor, $R$ the distance of shells in $\AA$.

\begin{tabular}{llll}
\hline sample 4 As: $R_{\mathrm{As}}, \sigma^{2}$ & $12 \mathrm{Ga}: R_{\mathrm{Ga}}, \sigma^{2}$ & $12 \mathrm{As}: R_{\mathrm{As}}, \sigma^{2}$ \\
\hline $500^{\circ} \mathrm{C}$ & $2.557(2), 0.0055(6)$ & $4.06(4), 0.025(6)$ & $4.73(3), 0.018(4)$ \\
$600^{\circ} \mathrm{C}$ & $2.577(4), 0.0049(2)$ & $4.23(4), 0.025(7)$ & $4.68(2), 0.017(3)$ \\
\hline
\end{tabular}

To the authors' knowledge the existence of cubic inclusions with $12 \mathrm{Mn}$ in the second shell was reported in none of the published EXAFS results (see [8] and references therein). The results of EXAFS analysis for samples annealed at $500{ }^{\circ} \mathrm{C}$ and $600{ }^{\circ} \mathrm{C}$ are presented in Fig. 4 and numerical results in Table 1.

One can notice the increase of the subsequent shells distance from $\mathrm{Mn}$ atoms in the sample annealed at $600{ }^{\circ} \mathrm{C}$. The considerable disorder in the Ga shell indicates that $\mathrm{Mn}$ atoms can be present in this shell. The best fit achieved under assumption of one $\mathrm{Mn}$ atom located in the second shell is presented in the inset of Fig. 4 for the sample annealed at $600{ }^{\circ} \mathrm{C}$. Such model is within the accuracy of EXAFS method providing slightly worse agreement with the experimental data and a quite large error in the parameters of the appropriate scattering path. This scattering path is located at the distance 4.43(16) $\AA$ with disorder factor $\sigma^{2}=0.007(6)$. The presence of $1 \mathrm{Mn}$ atom in a second shell gives in a $6 \mathrm{~nm}$ cluster the concentration of Mn close to $20 \%$. The characteristic feature of the investigated sample was that in the as grown layer $90 \%$ of Mn atoms occupied the substitutional position as was reported in Ref. [9] from a study performed for the same sample. It implies that the substitutional position of $\mathrm{Mn}$ atoms is stable and for producing hexagonal MnAs inclusions in such a layer an annealing temperature higher than $600{ }^{\circ} \mathrm{C}$ is necessary.

In Fig. 5, the Curie temperatures as a function of $\mathrm{Mn}$ concentration in $\mathrm{ZB}$ GaMnAs solid solution were calculated by using the hybrid method [10]. Effective exchange interactions between $\mathrm{Mn}$ atoms were estimated from firstprinciples and Curie temperatures were calculated by performing Monte Carlo simulations.

The LDA $+U$ method was employed to describe the electronic structure of GaMnAs correctly [10]. The agreement between the theoretical predictions and available ex-

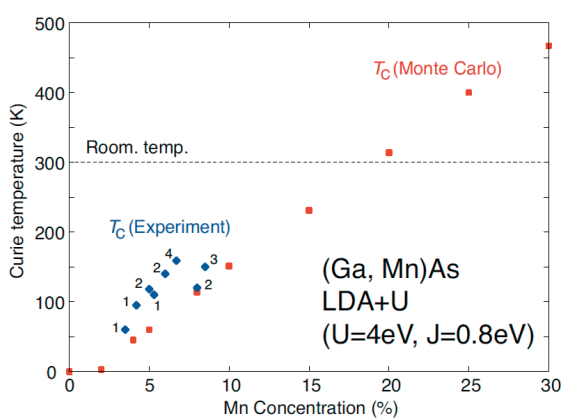

Figure 5 (online colour at: www.pss-rapid.com) Dependence of Curie temperature on the content of $\mathrm{Mn}$ in GaMnAs solid solution as result from calculation (squares). Available experimental values [11] are plotted by diamonds. perimental Curie temperatures is good, knowing that the experimental determination of $\mathrm{Mn}$ content is difficult. One can notice that the content of Mn close to $20 \%$ is sufficient to increase the Curie temperature to $300 \mathrm{~K}$. Observation of ferromagnetic-like behavior at temperatures as high as $295 \mathrm{~K}$ suggests that at least some clusters must contain about $20 \%$ of $\mathrm{Mn}$. There are probably also clusters with lower Mn content, which are paramagnetic at $295 \mathrm{~K}$, but are ferromagnetic at lower temperatures (e.g. clusters with $6-7 \%$ of $\mathrm{Mn}$ are ferromagnetic below about $150 \mathrm{~K}$, see Fig. 5). These clusters may be responsible for increase of the saturation value of magnetization at low temperatures, where all clusters (both with low and high Mn content) contribute to saturated magnetization. We estimated that about $80 \%$ of $\mathrm{Mn}$ ions enter highly concentrated ( $20 \%$ $\mathrm{Mn})$ clusters.

Concluding, the presently reported studies revealed that the source of room temperature ferromagnetism in granular layers with cubic $(\mathrm{Mn}, \mathrm{Ga}) \mathrm{As}$ clusters embedded in GaAs matrix are small (from $3 \mathrm{~nm}$ to $6 \mathrm{~nm}$ ) $\mathrm{ZB}$ inclusions. These inclusions are composed not of ZB MnAs but of GaMnAs solid solution with content of Mn much higher than reported in the literature for layers so far.

Acknowledgements This work was partially supported by national grant of Ministry of Science and High Education N202$052-32 / 1189$. The measurements performed at synchrotron have received funding from the European Community's Seventh Framework Programme (FP7/2007-2013) under grant agreement $n^{\circ} 226716$

\section{References}

[1] P. N. Hai, S. Ohya, M. Tanaka, S. E. Barnes, and S. Maekawa, Nature 458, 489 (2009).

[2] M. Yokoyama, H. Yamaguchi, T. Ogawa, and M. Tanaka, J. Appl. Phys. 97, 10D317-1 (2005).

[3] A. Kwiatkowski, D. Wasik, M. Kamńska, R. Bożek, J. Szczytko, A. Twardowski, J. Borysiuk, J. Sadowski, and J. Gosk, J. Appl. Phys. 101, 113912 (2007).

[4] A. Chanda, H. P. Lenka, and Ch. Jacob, Appl. Phys. A 94, 89 (2009).

[5] M. Moreno, J. I. Cerdá, K. H. Ploog, and K. Horn, Phys. Rev. B 82, 045117 (2010).

[6] R. Shioda, K. Ando, T. Hayashi, and M. Tanaka, Phys. Rev. B 58, 1100 (1998).

[7] B. Ravel and M. Newville, J. Synchrotron Radiat. 12, 537 (2005).

[8] I. N. Demchenko, K. Lawniczak-Jablonska, T. Story, V. Osinny, R. Jakiela, J. Z. Domagala, J. Sadowski, M. Klepka, A. Wolska, and M. Chernyshova, J. Phys.: Condens. Matter 19, 496205 (2007).

[9] K. Lawniczak-Jablonska, J. Libera, A. Wolska, M. T. Klepka, R. Jakiela, and J. Sadowski, Radiat. Phys. Chem. 78, S80 (2009).

[10] K. Sato et al., Rev. Mod. Phys. 82, 1633 (2010).

[11] F. Matsukura et al., Phys. Rev. B 57, R2037 (1998). K. W. Edmonds et al., Appl. Phys. Lett. 81, 4991 (2002).

K. C. Ku et al., Appl. Phys. Lett. 82, 2302 (2003).

K. W. Edmonds et al., Phys. Rev. Lett. 92, 37201 (2004). 